\title{
Telepathology: is there any future for this technique?
}

\author{
Wojciech Biernat
}

Telepathology is a branch of telemedicine. Its advances in recent years have raised several questions concerning its usage and problems it poses. These issues are shown in this paper.

Key words: telepathology, pathology

Telepathology is a subspecialty of telemedicine. It is a histopathological diagnostic procedure that is based on the analysis of digital images displayed on a screen in contrast to the conventional pathological study of slides under a light microscope [1]. The images are acquired by special scanners and send to other remote sites (even within the same unit). The earliest attempt to perform such a diagnosis remotely was undertaken in 1959 and concerned the transfer of radiological images. Still microscopic images may be captured and transmitted (static telepathology). Modern techniques allow transmission of real-time images from the light microscope, a method under the control of a pathologist at a remote site (dynamic telepathology). Some systems combine both techniques (hybrid systems).

In 1973, an incidental application of static telepathology made possible the diagnosis of mediastinal lymphoma in a patient in Brasil by a team of hematologists in Washington DC from bone marrow aspiration smears. Systemic introduction of telepathology into everyday practice started in Norway in 1990. It made pathological diagnosis feasible (mostly intraoperative) in the hospitals distributed over the sparsely populated northern parts of this country [1]. All these examples raise issues of the potential of extensive application of telepathology and the problems it brings about.

Intraoperative diagnosis seems to be the most appropriate application of telepathology. It requires immediate decisions concerning vital therapeutic issues that must be rendered in a relatively short time. As the availability of specialists may be limited due to the shortage of pathologists in general, especially in some sparsely populated geographic regions, transmission of microscopic images from the intra- operative tissue material may supplement these needs and allow proper management of the patients [1].

This technique may be used not only in primary diagnostics performed intraoperatively, but on routinely processed (formalin-fixed, parafin embedded, FFPE) material as well. An example of this approach was described in the case of remote consultations on pediatric oncological lesions in patients of the Queen Elisabeth Hospital w Blantyre (Malawi) and pediatric hospitals in Great Britain [3]. Of the 127 samples in which local and remote pathology were available, 81 (64\%) were concordant in their diagnosis, in 38 (30\%) a diagnosis was made by one centre (and none in the other) and $8(6 \%)$ had conflicting diagnoses.

Primary diagnostics may also concern the determination of predictive factors, i.e., HER2 expression in breast carcinoma. It is cost-effective but requires expensive hardware that allows digitalization of the microscopic slides. However, the cost of this equipment is decreasing and technological advances make scanning the slides possible even by means of simple adapters that may directly attach a smartphone to a microscope ocular [4]. A still important and limiting factor is the time required for scanning and transmission that remains problematic in some geographic regions

Most pathologists suggest that telepathology is playing a more important role in secondary diagnostics, i.e. expert consultations [2]. The time factor is decreasing in comparison to the conventional manner of verification of pathological diagnoses in another centre on condition that it does not require additional immunohistochemical and/ or molecular analyses. In these instances, transfer of tissue is indispensable for more a precise and final diagnosis due 
to the ability to implement methods not available in the primary centre. Of course, transfer of digital slides is more advantageous as it obviates the potential loss of samples in the conventional sending of blocks and slides by mail [2]. Most important of all is the potential of acquiring a highly expert opinion from people or centres dealing with specific subspecialities in pathology (e.g. dermatopathology, soft tissue pathology, diseases of the central nervous system, etc.) [5]

An important advantage of telepathology is the high concordance of diagnoses. The agreement between the telepathology and glass slide diagnoses approaches 80$100 \%$ and concerns both frozen section diagnosis and FFPE material $[1,6]$.

Last but not least, telepathology is extensively used in pathology education. Expanding banks of microscopic slides and digital gross images are valuable sources for teaching students and pathologists. They may be available both during courses and at home, increasing vastly the recognition of common diseases and unusual cases.

There are some areas in which telepathology brings about problems: technological (speed of scanning, transfer, etc.), economic (cost of scanners, high-efficient transfer devices), psychological (pathologists are used to perform diagnosis in the light microscopy) and medicolegal (responsibility for the proper and optimal selection of gross sectioning tissue later diagnosed by pathologists evaluating microscopic slides only). Solving the former aspects is associated with technological advances; it also influences the continuous decrease of costs. These aspects are reflected in the official reports of the COST agency (European Cooperation in the field of Scientific and Technical Research) whose analyses include the adequacy of current existing technical solutions [7].

The psychological aspects of telepathology are perhaps most difficult to overcome. The surveys of opinions in the pathological community show that the main task for this method is perceived with teaching programmes (48\%) [8]. Although $82 \%$ of pathologists regarded it helpful in obta- ining secondary opinion, $74 \%$ found sending slides and blocks by conventional mail the most appropriate method of consultation [8]. However, the changes that took place in radiological diagnostics in the 1990s and the shift from radiographs or radiological plates to digital images prove that this scenario is realistic in the everyday practice of pathologists.

\section{Conflict of interest: none declared}

\section{prof. Wojciech Biernat, MD, PhD}

Medical University of Gdańsk

Department of Pathomorphology

ul. Mariana Smoluchowskiego 17

80-214 Gdańsk, Poland

e-mail:wojciech.biernat@gumed.edu.pl

Received \& Accepted: 18 Dec 2017

Based on the presentation at the V Annual Conference of the Nowotwory Journal of Oncology, 'Oncological Debates', held in Warszawa, 7-8th April 2017

\section{References}

1. Cross SS, Dennis T, Start RD. Telepathology: current status and future prospects in diagnostic histopathology. Histopathology 2002; 41: 91-109.

2. Cornish TC, Swapp RE, Kaplan KJ. Whole-slide imaging: routine pathologic diagnosis. Adv Anat Pathol 2012; 19: 152-159.

3. Carey P, Fudzulani R, Scholfield D et al. Remote and rapid pathological diagnosis in a resource challenged unit. J Clin Pathol 2014; 67: 540-543.

4. Hartman DJ. Mobile technology for the practice of pathology. Adv Anat Pathol 2016; 23: 118-124.

5. Zembowicz A, Ahmad A, Lyle SR. A comprehensive analysis of a web-based dermatopathology second opinion consultation practice. Arch Pathol Lab Med 2011; 135: 379-383.

6. Chorneyko K, Giesler R, Sabatino D et al. Telepathology for routine light microscopic and frozen section diagnosis. Am J Clin Pathol 2002; 117: 783-790.

7. Garcia-Rojo M, Goncalves L, Blobel B. The COST Action IC0604 "Telepathology Network in Europe" (EURO-TELEPATH). Stud Health Technol Inform 2012; 179: 3-12.

8. Chordia TD, Vikey A, Choudhary $A B$ et al. Current status and future trends in telepathology and digital pathology. J Oral Maxillofac Pathol 2016; 20: 178-182. 